\title{
Quadratic Speed-Up for Simulating Gaussian Boson Sampling
}

\author{
Nicolás Quesada, ${ }^{1,2, *, \dagger}$ Rachel S. Chadwick,${ }^{3,4, \dagger}$ Bryn A. Bell,,${ }^{5, \dagger}$ Juan Miguel Arrazola, ${ }^{1}$ \\ Trevor Vincent, ${ }^{1}$ Haoyu Qi, ${ }^{1}$ and Raúl García-Patrón ${ }^{6}$ \\ ${ }^{1}$ Xanadu, 777 Bay Street, Toronto, Ontario M5G 2C8, Canada \\ ${ }^{2}$ Department of Engineering Physics, École Polytechnique de Montréal, Montréal, Québec H3T 1JK, Canada \\ ${ }^{3}$ Quantum Engineering Technology Labs, University of Bristol, Bristol BS8 1FD, United Kingdom \\ ${ }^{4}$ Quantum Engineering Centre for Doctoral Training, University of Bristol, Bristol BS8 1FD, United Kingdom \\ ${ }^{5}$ Department of Physics, Imperial College London, Ultrafast Quantum Optics group, London SW7 2AZ, United \\ Kingdom \\ ${ }^{6}$ School of Informatics, University of Edinburgh, Edinburgh EH8 9AB, United Kingdom
}

(Received 23 February 2021; accepted 8 November 2021; published 11 January 2022)

\begin{abstract}
We introduce an algorithm for the classical simulation of Gaussian boson sampling that is quadratically faster than previously known methods. The complexity of the algorithm is exponential in the number of photon pairs detected, not the number of photons, and is directly proportional to the time required to calculate a probability amplitude for a pure Gaussian state. The main innovation is to use auxiliary conditioning variables to reduce the problem of sampling to the computation of the pure-state probability amplitudes, for which the most computationally expensive step is the calculation of a loop hafnian. We implement and benchmark an improved loop-hafnian algorithm and show that it can be used to compute pure-state probabilities, the dominant step in the sampling algorithm, of events involving up to 50 photons in a single workstation, i.e., without the need of a supercomputer.
\end{abstract}

DOI: 10.1103/PRXQuantum.3.010306

\section{INTRODUCTION}

The building of quantum computers capable of convincingly performing tasks that are intractable to replicate using classical computers is a significant technological milestone $[1,2]$. For photonic implementations, boson sampling and its variants are a leading approach for demonstrating such capabilities [3-10]. Gaussian boson sampling (GBS) has emerged as a platform to tackle challenges in scaling to larger numbers of photons and modes [11, 12]. GBS has already been experimentally implemented [13-18], and it has generated additional interest due to the discovery of algorithms and applications [19-26]. These advances make GBS a leading candidate for demonstrating a quantum advantage in photonics.

In addition to experimental implementations, significant progress has also been made in developing classical simulation algorithms. For boson sampling, an early method

*nicolas.quesada@polymtl.ca

$\dagger$ These authors contributed equally.

Published by the American Physical Society under the terms of the Creative Commons Attribution 4.0 International license. Further distribution of this work must maintain attribution to the author(s) and the published article's title, journal citation, and DOI. is an approximate Markov-chain Monte Carlo algorithm [27]. This has been improved in Ref. [28], where an exact sampling algorithm has been introduced such that the complexity for generating one sample with $N$ photons is equivalent to that of the calculation of an output probability amplitude. This, in turn, is equivalent to the computation of the permanent of an $N \times N$ matrix, which requires $O\left(N 2^{N}\right)$ time using the best-known methods [29].

An effort to obtain simulation techniques for GBS has also been pursued. An exact algorithm has been reported and implemented for GBS with threshold detectors $[30,31]$ but it suffers from exponential memory requirements. Two algorithms have also been proposed in Ref. [32] for a restricted version of GBS. The first has polynomial space complexity and $O\left(\operatorname{poly}(N) 2^{8 N / 3}\right)$ time complexity; the second has exponential space complexity and $O\left(\operatorname{poly}(N) 2^{5 N / 2}\right)$ time complexity. Recently, an exact sampling algorithm for GBS has been presented that requires only polynomial memory [33]. This algorithm shows an improved complexity proportional to $O\left(N^{3} 2^{N}\right)$ for generating one sample with $N$ photons for both pure and mixed states. These GBS algorithms mark a crucial difference with respect to boson sampling: in GBS with pure states, the probability of observing an outcome with $N$ photons is proportional to the loop hafnian of an $N \times N$ matrix, for which the best algorithms require $O\left(N^{3} 2^{N / 2}\right)$ time. In other 
words, there is a quadratic gap between the complexity to generate a sample and the complexity to compute an output probability. This suggests the possible existence of a better sampling algorithm with complexity matching that of the calculation of an output probability amplitude, similar to what has been achieved for boson sampling.

We present such an algorithm. The main insight is to introduce conditioning auxiliary variables obtained by performing a virtual heterodyne measurement in all modes and iteratively replace the heterodyne outcomes with photon-number measurements. The photon-number outcomes conditional on the heterodyne measurements are given by pure-state probabilities. We develop a chain of conditional probabilities, where at each step only probabilities of a pure Gaussian state need to be calculated. For outputs with $N$ photons, these are proportional to loop hafnians of $N \times N$ matrices, which can be calculated in $O\left(N^{3} 2^{N / 2}\right)$ time [34]. In general, the number of output probabilities that must be calculated is proportional to the number of modes $m$, which leads to a time complexity upper bounded by $O\left(m N^{3} 2^{N / 2}\right)$. This corresponds to a quadratic speed-up over the previous state of the art. It also suggests that, compared to boson sampling, roughly twice as many photons are needed in GBS to reach the regime where classical simulations become intractable. We implement an improved version of a loop-hafnian algorithm and use it to compute pure-state probabilities of events with up to 50 photons using a workstation with 96 CPUs.

In what follows, we begin by giving a short overview of GBS in Sec. II. We then describe our simulation algorithm in Sec. III, which constitutes our main result. In Sec. IV, we benchmark an algorithm for the computation of loop hafnians, which determine the most expensive step for simulation, and finally we present a discussion of our findings in Sec. V.

\section{GAUSSIAN BOSON SAMPLING}

The quantum state of a collection of $m$ optical modes can be uniquely described in terms of its Wigner function $[35,36]$. Gaussian states are states the Wigner function of which is a Gaussian distribution. They can be described by a covariance matrix $\mathbf{V}$ and a vector of means $\overline{\mathbf{R}}=\left[\begin{array}{l}\overline{\mathbf{q}} \\ \overline{\mathbf{p}}\end{array}\right]$, where $\overline{\mathbf{q}}, \overline{\mathbf{p}}$ are the mean canonical position and momentum vectors. It is also possible to express the covariance matrix in terms of the complex amplitudes $\boldsymbol{\alpha}=(1 / \sqrt{2 \hbar})(\mathbf{q}+$ ip) $\in \mathbb{C}^{m}$, which are described by a complex-normal distribution with mean $\overline{\boldsymbol{\alpha}}=(1 / \sqrt{2 \hbar})(\overline{\mathbf{q}}+i \overline{\mathbf{p}}) \in \mathbb{C}^{m}$ and covariance matrix [37],

$$
\boldsymbol{\Sigma}=\mathbf{F V F}^{\dagger}, \quad \mathbf{F}:=\frac{1}{\sqrt{2 \hbar}}\left[\begin{array}{cc}
\mathbb{I} & i \mathbb{I} \\
\mathbb{I} & -i \mathbb{I}
\end{array}\right]
$$

where $\mathbb{I}$ is the identity matrix.
GBS is a form of photonic quantum computing where a Gaussian state is measured in the photon-number basis. If $\bar{\alpha}=0$, the probability of observing the output sample $S=$ $\left(s_{1}, \ldots, s_{m}\right)$, where $s_{i}$ is the number of photons in mode $i$, is given by [11]

$$
p(S)=\frac{1}{\sqrt{\operatorname{det}(\mathbf{Q})}} \frac{\operatorname{haf}\left(\mathbf{A}_{S}\right)}{s_{1} ! \cdots s_{m} !},
$$

where

$$
\begin{gathered}
\mathbf{Q}:=\Sigma+\mathbb{I} / 2, \\
\mathbf{A}:=\mathbf{X}\left(\mathbb{I}-\mathbf{Q}^{-1}\right), \\
\mathbf{X}:=\left[\begin{array}{ll}
0 & \mathbb{I} \\
\mathbb{I} & 0
\end{array}\right]
\end{gathered}
$$

and $\mathbf{A}_{S}$ is the matrix obtained as follows: if $s_{i}=0$, rows and columns $i$ and $i+m$ are deleted from $\mathbf{A}$; if $s_{i}>0$, the rows and columns are repeated $s_{i}$ times. The hafnian of a square symmetric matrix of even dimension $n$ is defined as

$$
\operatorname{haf}(\mathbf{C})=\sum_{X \in \operatorname{PMP}(n)} \prod_{(i, j) \in X} \mathbf{C}_{i, j},
$$

where $\operatorname{PMP}(n)$ is the set of perfect matching permutations [34].

Defining $N:=\sum_{i} s_{i}$ as the total number of photons, the submatrix $\mathbf{A}_{S}$ has dimension $2 N$, meaning that the best algorithm for the calculation of its hafnian requires $O\left(N^{3} 2^{2 N / 2}\right)=O\left(N^{3} 2^{N}\right)$ time. However, when the Gaussian state is pure, it is possible to write $\mathbf{A}=\mathbf{B} \oplus \mathbf{B}^{*}$ and the probability distribution reduces to [11]

$$
p(S)=\frac{1}{\sqrt{\operatorname{det}(\mathbf{Q})}} \frac{\left|\operatorname{haf}\left(\mathbf{B}_{S}\right)\right|^{2}}{s_{1} ! \cdots s_{m} !},
$$

where $\mathbf{B}_{S}$ is constructed analogously: if $s_{i}=0$, rows and columns $i$ are deleted; if $s_{i}>0$, the rows and columns are repeated $s_{i}$ times. Since the matrix $\mathbf{B}_{S}$ has dimension $N$, computation of its hafnian requires only $O\left(N^{3} 2^{N / 2}\right)$ time.

An analogous formula can also be derived for GBS with displacements, i.e., when $\overline{\boldsymbol{\alpha}} \neq 0$. We define the following quantities:

$$
\begin{gathered}
\vec{\alpha}=\left[\begin{array}{c}
\overline{\boldsymbol{\alpha}} \\
\overline{\boldsymbol{\alpha}}^{*}
\end{array}\right]=\mathbf{F} \overline{\mathbf{R}}, \\
\vec{\gamma}=\left[\begin{array}{c}
\bar{\gamma} \\
\bar{\gamma}^{*}
\end{array}\right]=\left(\mathbf{Q}^{-1} \vec{\alpha}\right)^{*},
\end{gathered}
$$




$$
p(\operatorname{vac})=\frac{\exp \left(-(1 / 2)[\vec{\alpha}]^{\dagger}[\mathbf{Q}]^{-1} \vec{\alpha}\right)}{\sqrt{\operatorname{det}(\mathbf{Q})}} .
$$

In this case, the output probabilities are given by $[34,38]$ :

$$
p(S)=p(\operatorname{vac}) \frac{\operatorname{lhaf}\left\{\text { filldiag }\left(\mathbf{A}_{S}, \vec{\gamma}_{S}\right)\right\}}{s_{1} ! \cdots s_{m} !},
$$

where lhaf(.) is the loop hafnian [34], $\vec{\gamma}_{S}$ is obtained from $\vec{\gamma}$ by repeating the $i, i+m$ entries of $\vec{\gamma}$ a total of $s_{i}$ times, and filldiag $\left(\mathbf{A}_{S}, \vec{\gamma}_{S}\right)$ replaces the diagonal of $\mathbf{A}_{S}$ with the vector $\vec{\gamma}_{S}$. The loop hafnian of an arbitrary matrix is defined analogously to the hafnian in Eq. (6) but replacing the set $\operatorname{PMP}(n)$ by the set of single-pair matchings $\operatorname{SPM}(n)$ [34].

When the Gaussian state is pure, it is possible to express the output probability as

$$
p(S)=p(\mathrm{vac}) \frac{\mid \text { lhaf }\left.\left\{\text { filldiag }\left(\mathbf{B}_{S}, \overline{\boldsymbol{\gamma}}_{S}\right)\right\}\right|^{2}}{s_{1} ! \cdots s_{m} !},
$$

where $\bar{\gamma}_{S}$ is obtained from $\bar{\gamma}$ by repeating its $i$ th entry $s_{i}$ times.

Up to constant prefactors, the best-known algorithms for the calculation of loop hafnians of generic matrices have the same complexity as for hafnians, namely $O\left(N^{3} 2^{N / 2}\right)$ for matrices of dimension $N$. This implies that the complexity of computing output probabilities with $N$ photons for pure Gaussian states with displacements also scales as $O\left(N^{3} 2^{N / 2}\right)$. We make use of this result in Sec. III.

So far, we have focused only on describing the photonnumber statistics of a multimode Gaussian state. For the sampling algorithm that we describe in Sec. III, it is also necessary to recall some basic properties of continuous-output measurements on a Gaussian state. Of particular relevance here are so-called heterodyne measurements. The outcomes of an $m$-mode heterodyne measurement are specified by a vector of complex numbers $\boldsymbol{\alpha}=(1 / \sqrt{2 \hbar})(\mathbf{q}+i \mathbf{p}) \in \mathbb{C}^{m}$. The generation of heterodyne samples from a Gaussian state is straightforward. For a Gaussian state with vector of means $\overline{\mathbf{R}}$ and covariance matrix $\mathbf{V}$, we sample from the multivariate normal distribution $\boldsymbol{\mu} \sim \mathcal{N}\left(\overline{\mathbf{R}}, \mathbf{V}_{Q}\right)$, where $\boldsymbol{\mu}=\left[\begin{array}{l}\mathbf{q} \\ \mathbf{p}\end{array}\right]$ and $\mathbf{V}_{Q}$ is the Husimi $Q$-function covariance matrix [39] in the quadrature basis, given by

$$
\mathbf{V}_{Q}=\mathbf{V}+\frac{\hbar}{2} \mathbb{I}
$$

Explicitly, we obtain outcome $\mu$ with probability

$$
p(\boldsymbol{\mu})=\frac{\exp \left(-(1 / 2)(\boldsymbol{\mu}-\overline{\mathbf{R}})^{T} \mathbf{V}_{Q}^{-1}(\boldsymbol{\mu}-\overline{\mathbf{R}})\right)}{\sqrt{\operatorname{det}\left(2 \pi \mathbf{V}_{Q}\right)}} .
$$

A partial heterodyne measurement can be performed when measuring only a subset of the modes. This can be done either by sampling from the reduced matrix of $\mathbf{V}_{Q}$, formed by selecting only the rows and columns of the included modes, or by sampling all modes and discarding the outcomes for modes we do not wish to sample.

It is also useful to write the conditional state of a subset of the modes, $\mathcal{A}$, when the other modes, $\mathcal{B}$, are measured using heterodyne detectors. For this, it is convenient to write the covariance matrix $\mathbf{V}$ in block form, with modes in $\mathcal{A}$ and $\mathcal{B}$ in separate blocks, and similarly to group the modes in the vector of means. This can be done by permuting the rows and columns in the covariance matrix and the elements of the vector of means, keeping the ordering in both consistent. So we write both the covariance matrix and the vector of means in the ordering $\left(\mathbf{q}_{\mathcal{A}}, \mathbf{p}_{\mathcal{A}}, \mathbf{q}_{\mathcal{B}}, \mathbf{p}_{\mathcal{B}}\right)$ :

$$
\mathbf{V}=\left(\begin{array}{cc}
\mathbf{V}_{\mathcal{A A}} & \mathbf{V}_{\mathcal{A B}} \\
\mathbf{V}_{\mathcal{B A}} & \mathbf{V}_{\mathcal{B B}}
\end{array}\right), \quad \overline{\mathbf{R}}=\left[\begin{array}{c}
\overline{\mathbf{R}}_{\mathcal{A}} \\
\overline{\mathbf{R}}_{\mathcal{B}}
\end{array}\right]
$$

If the outcome $\boldsymbol{\mu}_{\mathcal{B}}$ is obtained by measuring the modes in the set $\mathcal{B}$, we can write the resulting covariance matrix and vector of means for $\mathcal{A}$ as [36]

$$
\begin{gathered}
\mathbf{V}_{\mathcal{A}}^{(\mathcal{B})}=\mathbf{V}_{\mathcal{A} \mathcal{A}}-\mathbf{V}_{\mathcal{A B}}\left(\mathbf{V}_{\mathcal{B B}}+\frac{\hbar}{2} \mathbb{I}\right)^{-1} \mathbf{V}_{\mathcal{B} \mathcal{A}}, \\
\mathbf{R}_{\mathcal{A}}^{(\mathcal{B})}=\overline{\mathbf{R}}_{\mathcal{A}}+\mathbf{V}_{\mathcal{A B}}\left(\mathbf{V}_{\mathcal{B B}}+\frac{\hbar}{2} \mathbb{I}\right)^{-1}\left(\boldsymbol{\mu}_{\mathcal{B}}-\overline{\mathbf{R}}_{\mathcal{B}}\right) .
\end{gathered}
$$

This update rule, together with the sampling in Eq. (14), is useful in the next section, where we introduce our algorithm.

\section{ALGORITHM}

We now describe an algorithm that samples the modes sequentially but that, unlike the one from Ref. [33], never requires the calculation of mixed-state probabilities. In this algorithm, we introduce partial heterodyne measurements so that only pure-state probabilities need to be evaluated at each step. Given a partial heterodyne measurement on modes in $\mathcal{B}$, the photon-number probability of a pattern $S$ in modes $\mathcal{A}$ is given by Eq. (12), where $\mathbf{B}$ and $\boldsymbol{\gamma}$ are now found for the state with covariance matrix $\mathbf{V}_{\mathcal{A}}^{(\mathcal{B})}$ and vector of means $\mathbf{R}_{\mathcal{A}}^{(\mathcal{B})}$ as given in Eq. (17). Note that if the global covariance matrix $\mathbf{V}$ corresponds to a pure state, the conditional covariance matrix $\mathbf{V}_{\mathcal{A}}^{(\mathcal{B})}$ also corresponds to a pure state.

If we wish to sample in the photon-number basis in modes $\mathcal{A}$ without measuring the other modes, we can make use of the observation in the paragraph above: the marginal photon-number probabilities can be obtained by integrating the joint probabilities over the set of possible 
heterodyne outcomes $\boldsymbol{\alpha}_{\mathcal{B}}$ :

$$
p\left(\mathbf{s}_{\mathcal{A}}\right)=\int d \boldsymbol{\alpha}_{\mathcal{B}} p\left(\mathbf{s}_{\mathcal{A}}, \boldsymbol{\alpha}_{\mathcal{B}}\right)=\int d \boldsymbol{\alpha}_{\mathcal{B}} p\left(\boldsymbol{\alpha}_{\mathcal{B}}\right) p\left(\mathbf{s}_{\mathcal{A}} \mid \boldsymbol{\alpha}_{\mathcal{B}}\right) .
$$

Hence we can sample from the marginal probabilities by sampling from $p\left(\boldsymbol{\alpha}_{\mathcal{B}}\right)$, followed by the conditional probabilities $p\left(\mathbf{s}_{\mathcal{A}} \mid \boldsymbol{\alpha}_{\mathcal{B}}\right)$ and then simply ignoring the heterodyne outcome. This is justified because one can always sample from a marginal distribution by considering additional virtual variables and then sampling from the correspondingly enlarged probability distribution, as long as one then forgets the values of the added variables. The outcomes of the heterodyne measurements are precisely these added variables; they do not correspond to real measurements in an experimental setup but are, rather, virtual measurements to introduce convenient conditioning auxiliary variables.

We now want to apply this directly to sampling the modes sequentially. The objective is to sample mode $k$ given that the previous modes $1, \ldots, k-1$ have already been sampled, so we set $\mathcal{A}=1, \ldots, k$ and $\mathcal{B}=k+$ $1, \ldots, m$. We assume that we have already sampled from $p\left(s_{1}, \ldots, s_{k-1}, \alpha_{k+1}, \ldots, \alpha_{m}\right)$. We wish to sample $s_{k}$ conditional on this outcome. To do this, we can calculate the relative probabilities for all $s_{k}$ and sample from that distribution. This results in a sample drawn from $p\left(s_{1}, \ldots, s_{k}, \alpha_{k+1}, \ldots, \alpha_{m}\right)$. By discarding $\alpha_{k+1}$, we are left with a sample from $p\left(s_{1}, \ldots, s_{k}, \alpha_{k+2}, \ldots, \alpha_{m}\right)$ and are ready to sample the $(k+1)$ th mode. We work progressively from $k=1$ to $m$, essentially replacing the virtual heterodyne measurements with photon-number measurements until we are left with a sample from $p\left(s_{1}, \ldots, s_{m}\right)$.

This algorithm can sample from a pure multimode state, yet in realistic experiments, the full state may not be pure; for example, if loss is included. To address this, we express the mixed Gaussian state as a convex combination of pure states. The Williamson decomposition $[35,40]$ of a quantum covariance matrix $\mathbf{V}$ states that it can be split as

$$
\mathbf{V}=\mathbf{T}+\mathbf{W},
$$

where $\mathbf{T}=(\hbar / 2) \mathbf{S S}^{T}$ is the covariance matrix of a pure state, $\mathbf{S}$ is a symplectic matrix, and $\mathbf{W}$ is positive semidefinite. In Hilbert space, this implies that a mixed Gaussian state with covariance matrix $\mathbf{V}=\mathbf{T}+\mathbf{W}$ and a vector of means $\overline{\mathbf{R}}=\left[\begin{array}{l}\overline{\mathbf{q}} \\ \overline{\mathbf{p}}\end{array}\right]$ can be expressed as $[36,41]$

$$
\varrho=\int d \mathbf{R} p(\mathbf{R})\left|\psi_{\mathbf{R}, \mathbf{T}}\right\rangle\left\langle\psi_{\mathbf{R}, \mathbf{T}}\right|,
$$

where

$$
p(\mathbf{R})=\frac{\exp \left(-(1 / 2)(\mathbf{R}-\overline{\mathbf{R}})^{T} \mathbf{W}^{-1}(\mathbf{R}-\overline{\mathbf{R}})\right)}{\sqrt{\operatorname{det}(2 \pi \mathbf{W})}},
$$

is the probability density function of a multivariate normal distribution and $\left|\psi_{\mathbf{R}, \mathbf{T}}\right\rangle$ is a pure Gaussian state with vector of means $\mathbf{R}$ and covariance matrix $\mathbf{T}$. Using this, the probability of observing an outcome $\left(s_{1}, s_{2}, \ldots, s_{m}\right)$ when performing a measurement on a mixed state $\varrho$ on $m$ modes is

$$
\begin{aligned}
p\left(s_{1}, \ldots, s_{m}\right) & =\left\langle s_{1}, \ldots, s_{m}|\varrho| s_{1}, \ldots, s_{m}\right\rangle \\
& =\int d \mathbf{R} p(\mathbf{R})\left|\left\langle\psi_{\mathbf{R}, \mathbf{T}} \mid s_{1}, \ldots, s_{m}\right\rangle\right|^{2} \\
& =\int d \mathbf{R} p(\mathbf{R}) p\left(s_{1}, \ldots, s_{m} \mid \mathbf{R}, \mathbf{T}\right) .
\end{aligned}
$$

Hence, to sample from a mixed state, we can sample the displacement vector from Eq. (21) and then sample from the resulting pure state.

We can now outline the full algorithm for simulating GBS.

Algorithm: To sample from a state given by covariance matrix $\mathbf{V}$ and vector of means $\overline{\mathbf{R}}$ in $m$ modes:

(1) If the Gaussian state to be sampled is mixed, calculate the matrices $\mathbf{T}, \mathbf{W}$ from the Williamson decomposition such that $\mathbf{V}=\mathbf{T}+\mathbf{W}$. Sample a vector $\mathbf{R}$ from the multivariate normal distribution $p(\mathbf{R})$ as in Eq. (21). This can be done in cubic time in the size of the matrix [42]. Continue the algorithm with the pure state given by covariance matrix $\mathbf{T}$ and vector of means $\mathbf{R}$.

(2) Generate a sample from the probability distribution $p\left(\alpha_{2}, \ldots, \alpha_{m}\right)$ resulting from heterodyne measurements on modes 2- $m$ using Eq. (14). This can be done in cubic time in the number of modes.

For modes $k=1, \ldots, m$ :

(3) Given the sample from $p\left(s_{1}, \ldots, s_{k-1}, \alpha_{k+1}, \ldots, \alpha_{m}\right)$ (which is found in the previous step), find $\mathbf{V}^{(k)}$ and $\mathbf{R}^{(k)}$ the covariance matrix and vector of means for the first $k$ modes conditional on the measurement $\left(\alpha_{k+1}, \ldots, \alpha_{m}\right)$ in the other modes, using Eqs. (16) and (17). For $k=1$, we start with a sample from $p\left(\alpha_{2}, \ldots, \alpha_{m}\right)$.

(4) Given a cutoff $d$, use Eq. (12) with $\mathbf{V}^{(k)}$ and $\mathbf{R}^{(k)}$ to compute the probabilities $p\left(s_{k}, s_{k-1}^{\star}, \ldots, s_{1}^{\star} \mid \alpha_{k+1}^{\star}\right.$, $\left.\ldots, \alpha_{m}^{\star}\right)$ for $s_{k}=0,1, \ldots, d$, with all starred variables sampled previously from the correct distribution. This is the most computationally demanding step, which requires the computation of loop hafnians.

(5) Sample $s_{k}$ with respect to the distribution

$$
\frac{p\left(s_{k}, s_{k-1}^{\star}, \ldots, s_{1}^{\star} \mid \alpha_{k+1}^{\star}, \ldots, \alpha_{m}^{\star}\right)}{\sum_{s_{k}} p\left(s_{k}, s_{k-1}^{\star}, \ldots, s_{1}^{\star} \mid \alpha_{k+1}^{\star}, \ldots, \alpha_{m}^{\star}\right)} .
$$




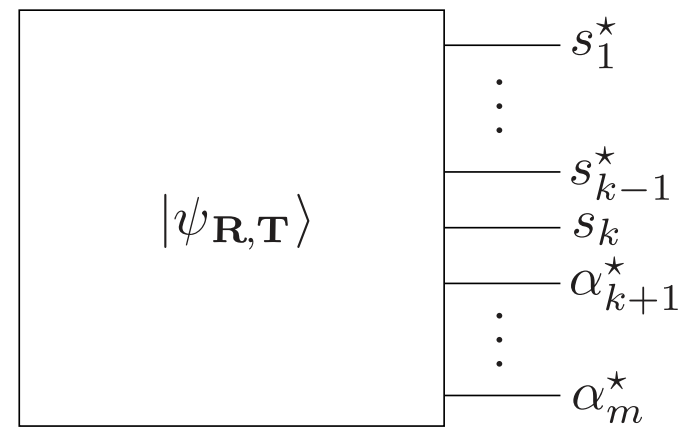

FIG. 1. In step $k$ of the algorithm, we calculate the relative probabilities of obtaining $s_{k}$ photons conditioned on the photonnumber outcomes of the previous modes and the heterodyne measurement outcomes of ensuing modes.

(6) Discard $\alpha_{k+1}^{\star}$ to obtain an outcome sampled from $p\left(s_{1}, \ldots, s_{k}, \alpha_{k+2}, \ldots, \alpha_{m}\right)$.

After repeating steps (3)-(6) for each $k=1,2, \ldots, m$, we are left with an outcome sampled from $p\left(s_{1}, \ldots, s_{m}\right)$. The algorithm is schematically represented in Fig. 1. Its correctness follows directly from the definition of conditional probabilities and integration over the auxiliary variables $\alpha_{2}, \ldots, \alpha_{m}$ as shown in the Appendix.

Overall, the algorithm reduces sampling from the GBS distribution to the computation of pure-state probabilities $p\left(s_{k}, s_{k-1}^{\star}, \ldots, s_{1}^{\star} \mid \alpha_{k+1}, \ldots, \alpha_{m}\right)$. When a total of $N$ photons are detected, calculation of the largest such probability amplitude requires $O\left(N^{3} 2^{N / 2}\right)$ time, which results from the computation of loop hafnians in step (4). This is scaled up by at most the total number of modes, giving a total sampling complexity of $O\left(m N^{3} 2^{N / 2}\right)$. This is a quadratic improvement over the algorithm in Ref. [33] which has complexity $O\left(m N^{3} 2^{N}\right)$.

It is worthwhile to compare to the algorithm of Ref. [28] for boson sampling, the complexity of which is $O\left(N 2^{N}\right)$. Up to polynomial factors, our algorithm suggests that the time required to simulate boson sampling with $N$ photons is roughly the same as simulating GBS with $2 N$ photons. We note that our algorithm can also be used to simulate GBS with threshold detectors: once a photon-number sample is generated, simply set $s_{i}=1$ if $s_{i}>0$, where $s_{i}=1$ denotes that the detector is measuring one or more photons.

Finally, note that each random variable $s_{k}$ has support over the non-negative integers; thus, in principle, one needs to calculate all the (infinitely many) conditional probabilities. However, we can choose some cutoff number of photons $d$ in each mode such that the probability of obtaining a photon number above this value is negligible. We confirm the accuracy of the algorithm in Fig. 2, where we show that the total variation distance lies within the expected range for the sample size as compared to a brute-force sampler with the same number of samples.

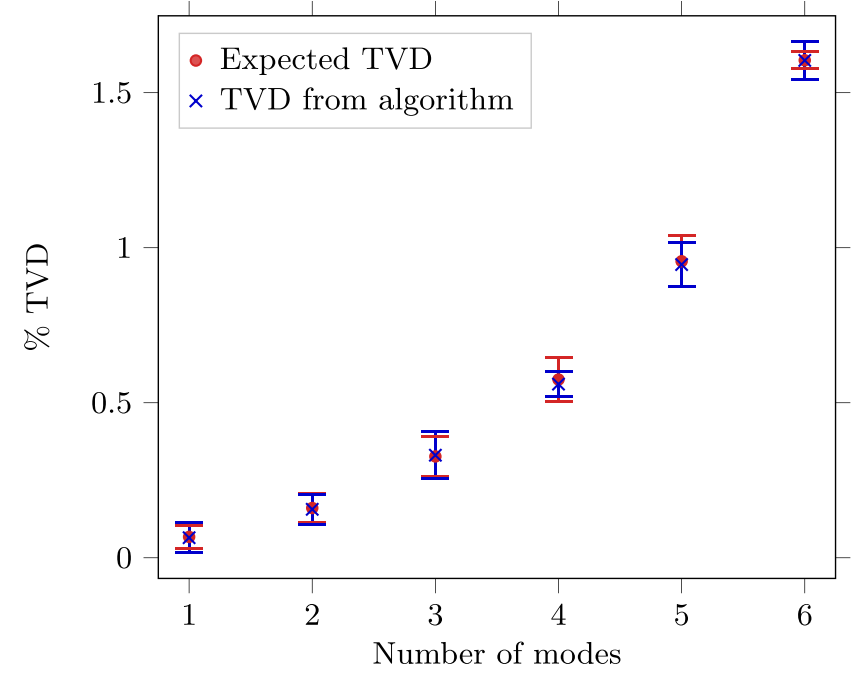

FIG. 2. The total variation distance (TVD) of the proposed algorithm and the expected TVD computed by estimating probabilities from brute-force sampling of the ideal distribution. We numerically find the TVD between the exact probabilities and the estimated probabilities for all outputs below the cutoff photon number. The expected TVD is estimated by sampling from the exact distribution. For both sampling algorithms, the probability distribution is estimated by taking a sample of size 200000 and averaging over ten Haar-random unitaries. The squeezing parameter is 0.5 in all modes, giving an average photon number of approximately 0.27 per mode, and we choose a cutoff of six photons. The error bars show the standard deviation of the ten repeated tests.

This shows that the chosen cutoff is sufficiently high not to introduce any notable error.

\section{BENCHMARKING}

In this section, we test the performance of an implementation of the loop-hafnian algorithm of Ref. [34], which is available in THE WALRUS [43]. The evaluation of loop hafnians is delegated to multithreaded $\mathrm{C}++$ code, which uses the La Budde algorithm [44] for the calculation of the characteristic polynomial of a matrix. This gives a speed-up of about three times with respect to previous algorithms based on diagonalization but, more importantly, significantly improves the accuracy of the calculation.

In the original implementation of the loop-hafnian algorithm, which uses double precision and eigenvalue methods for the calculation of power traces, it is found that a relative error of approximately $10^{-1}$ is present for the computation of the loop hafnian of the $54 \times 54$ all-ones matrix [34]. To get around this issue, we use the aforementioned La Budde algorithm to significantly improve the accuracy of the calculation of the characteristic polynomial of a matrix [44]. Moreover, this method allows us to use long double-complex data types. With these changes, we lower the relative error in the calculation by 3 orders of 


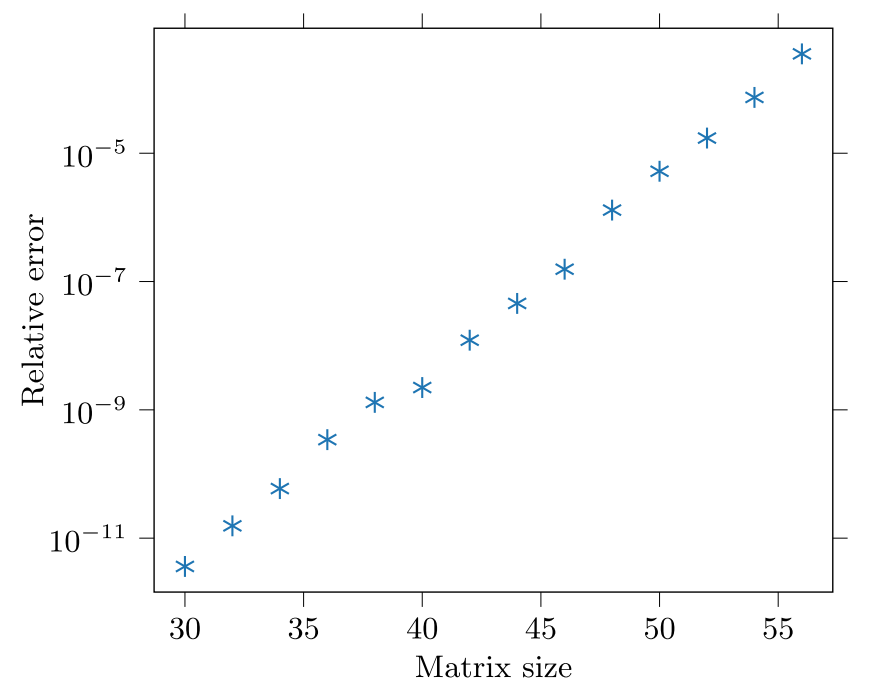

FIG. 3. The relative error in the calculation of the all-ones matrix $\mathbb{1}_{n}$ for different matrix sizes $n$. The exact value of the loop hafnian for a given size $n$ is given by the $n$th telephone number $T(n)$ [34]; the relative error is given by $\mid \operatorname{lhaf}_{\text {numeric }}\left(\mathbb{1}_{n}\right)-$ $T(n) \mid / T(n)$.

magnitude compared to the previous implementation. We can then achieve a precision of one part in 10000 for the computation of loop hafnians of matrices with dimension 56, as shown in Fig. 3.

As shown in the previous section, the run time of the algorithm scales exponentially with the number of photons and linearly with the number of modes. Since the number of photons is the dominant parameter, we benchmark the time taken to calculate the largest event. If $N$ photons are detected at the end of the algorithm, probabilities having at most $N+d$ photons need to be calculated, where $d$ is the cutoff.

In Fig. 4, we benchmark the time it takes to calculate the loop hafnian of a random symmetric complex matrix in long double-complex precision up to a total dimension of 56, corresponding to detection of $N=50$ photons with a cutoff of $d=6$. The computations are done on a workstation of 96 CPUs. The matrices are built by sampling each real and imaginary component from the standard uniform distribution and are then symmetrized. As can be deduced from the figure, the run time for the calculation of the loop hafnian of a $56 \times 56$ matrix is approximately $7 \mathrm{~h}$ and scales exponentially with the number of photons, which is reflected in the linear growth in logarithmic scale. We can estimate the total run time of the sampling algorithm by approximating the number of times that such loop hafnians need to be computed.

For a physical system with $m$ modes, an upper bound on the run time can be obtained by multiplying the run time of the largest loop-hafnian calculation by $m$. However, typically, the $N$ photons occur spread out across different modes, so we only calculate the largest loop hafnians for a

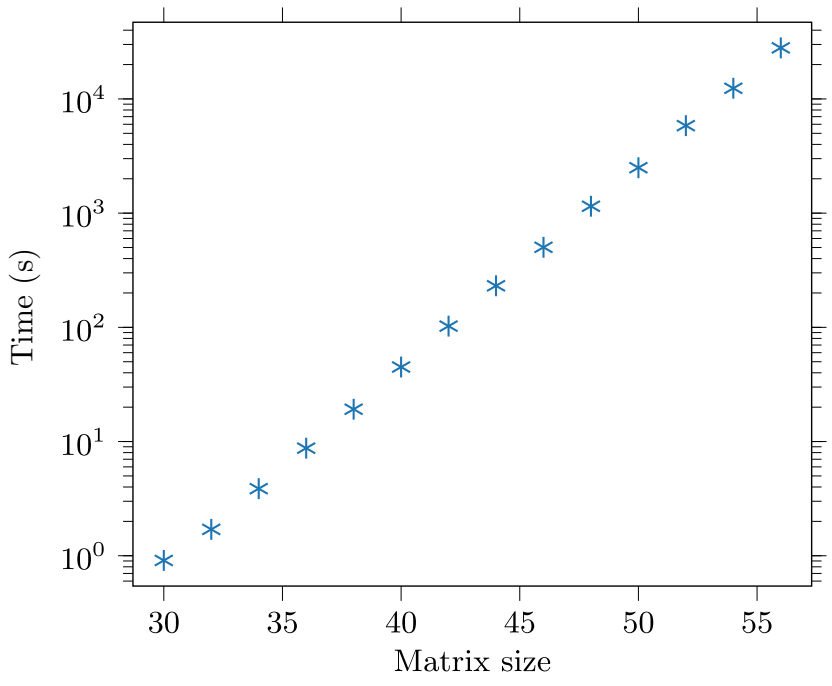

FIG. 4. The computation time of the loop hafnian of a random complex symmetric matrix in long double-complex precision using a Standard D96a v4 Microsoft Azure instance with 96 CPUs running at $2.345 \mathrm{GHz}$. For a matrix of dimension 56, the run time is approximately $7 \mathrm{~h}$.

fraction of the modes. The worst case occurs when photons are detected in the first $N$ modes, resulting in expensive calculations in the remaining $m-N$ modes. For example, for $m=100$ and $N=50$, we can estimate a run time of roughly 2 weeks for generating a single sample on the workstation.

Finally, the loop-hafnian implementation benchmarked here is written for portability and reproducibility. This implies that our implementation of the algorithm is not highly optimized. In the future, we expect to achieve a speed increase of 1-2 orders of magnitude by low-level optimization of the $\mathrm{C}++$ back end coupled with the use of a modern task-based parallelism library [45] for more efficient load-balancing.

\section{DISCUSSION}

We introduce an algorithm for the simulation of GBS for which the complexity of generating a sample scales, up to polynomial prefactors, like the complexity of calculating a probability amplitude. This results in a quadratic speed-up compared to the previous state of the art. The algorithm is exact up to a small error induced by a cutoff dimension, runs in polynomial space, and simulates the most general forms of GBS.

A remarkable consequence of our result is that GBS requires roughly twice as many photons than standard boson sampling to reach the same regime of classical simulation. This has potential implications for experimental efforts at demonstrating an advantage over classical simulators. Indeed, for a number of photons $N$ and a number of modes $m$, the complexity of the best algorithm for boson 
sampling scales as $O\left(N 2^{N}\right)$, whereas our algorithm has run time $O\left(m N^{3} 2^{N / 2}\right)$. A possible interpretation is that in GBS, where photons are generated through squeezing, it is the number of photon pairs that determine complexity, not the number of photons.

\section{ACKNOWLEDGMENTS}

N.Q. thanks L. Banchi, K. K. Sabapathy, and D. Schmid for fruitful discussions. N.Q. and T.V. thank A. Fumagalli, and J. Izaac for technical assistance. We thank I. Dhand and O. Di Matteo for comments on the manuscript. B.A.B. and R.S.C. thank J. F. F. Bulmer, A. E. Jones,
S. Paesani, and R. B. Patel for useful discussions. R.S.C. acknowledges support from the Engineering and Physical Sciences Research Council (Grant No. EP/LO15730/1). B.A.B. is supported by a European Commission Marie Skłodowska Curie Individual Fellowship (Frequencyencoded quantum multi-photon interference devices, Grant No. 846073).

\section{APPENDIX: PROOF OF CORRECTNESS}

We prove that the algorithm presented in Sec. III correctly samples from $p\left(s_{1}, \ldots, s_{m}\right)$. The distribution that we are sampling from is given by

$$
\begin{aligned}
\tilde{p}\left(s_{1}, \ldots, s_{m}\right) & =\int d \alpha_{2} \cdots d \alpha_{m} p\left(\alpha_{2}, \ldots, \alpha_{m}\right) p\left(s_{1} \mid \alpha_{2}, \ldots, \alpha_{m}\right) p\left(s_{2} \mid s_{1}, \alpha_{3}, \ldots, \alpha_{m}\right) \cdots p\left(s_{m} \mid s_{1}, \ldots, s_{m-1}\right) \\
& =\int d \alpha_{2} \cdots d \alpha_{m} p\left(\alpha_{2}, \ldots, \alpha_{m}\right) \frac{p\left(s_{1}, \alpha_{2}, \ldots, \alpha_{m}\right)}{p\left(\alpha_{2}, \ldots, \alpha_{m}\right)} \frac{p\left(s_{1}, s_{2}, \alpha_{3}, \ldots, \alpha_{m}\right)}{p\left(s_{1}, \alpha_{3}, \ldots, \alpha_{m}\right)} \cdots \frac{p\left(s_{1}, \ldots, s_{m}\right)}{p\left(s_{1}, \ldots, s_{m-1}\right)} \\
& =\int d \alpha_{2} \cdots d \alpha_{m} \frac{p\left(s_{1}, \alpha_{2}, \ldots, \alpha_{m}\right)}{p\left(s_{1}, \alpha_{3}, \ldots, \alpha_{m}\right)} \frac{p\left(s_{1}, s_{2}, \alpha_{3}, \ldots, \alpha_{m}\right)}{p\left(s_{1}, s_{2}, \alpha_{4}, \ldots, \alpha_{m}\right)} \cdots \frac{p\left(s_{1}, \ldots, s_{m-1}, \alpha_{m}\right)}{p\left(s_{1}, \ldots, s_{m-1}\right)} p\left(s_{1}, \ldots, s_{m}\right) \\
& =\int d \alpha_{2} \cdots d \alpha_{m} p\left(\alpha_{2} \mid s_{1}, \alpha_{3}, \ldots, \alpha_{m}\right) p\left(\alpha_{3} \mid s_{1}, s_{2}, \alpha_{4}, \ldots, \alpha_{m}\right) \cdots p\left(\alpha_{m} \mid s_{1}, \ldots, s_{m-1}\right) p\left(s_{1}, \ldots, s_{m}\right) \\
& =p\left(s_{1}, \ldots, s_{m}\right),
\end{aligned}
$$

which is precisely the GBS distribution. To go from the first to the second line, we use the definition of conditional probabilities. From the second to the third line, we rearrange the numerators and denominators of the different fractions. To arrive at the fourth line, we use the definition of conditional probability once more. In the last line, we use the fact $\int d \alpha_{k} p\left(\alpha_{k} \mid s_{1}, \ldots, s_{k-1}, \alpha_{k+1}, \ldots, \alpha_{m}\right)=1$ and integrate in order from $\alpha_{2}$ to $\alpha_{m}$.

[1] F. Arute, K. Arya, R. Babbush, D. Bacon, J. C. Bardin, R. Barends, R. Biswas, S. Boixo, F. G. Brandao, D. A. Buell, et al., Quantum supremacy using a programmable superconducting processor, Nature 574, 505 (2019).

[2] A. W. Harrow and A. Montanaro, Quantum computational supremacy, Nature 549, 203 (2017).

[3] S. Aaronson and A. Arkhipov, in Proceedings of the FortyThird Annual ACM Symposium on Theory of Computing (ACM, New York, NY, USA, 2011), p. 333.

[4] S. Barkhofen, T. J. Bartley, L. Sansoni, R. Kruse, C. S. Hamilton, I. Jex, and C. Silberhorn, Driven Boson Sampling, Phys. Rev. Lett. 118, 020502 (2017).

[5] A. P. Lund, A. Laing, S. Rahimi-Keshari, T. Rudolph, J. L. O'Brien, and T. C. Ralph, Boson Sampling from a Gaussian State, Phys. Rev. Lett. 113, 100502 (2014).
[6] A. P. Lund, S. Rahimi-Keshari, and T. C. Ralph, Exact boson sampling using Gaussian continuous-variable measurements, Phys. Rev. A 96, 022301 (2017).

[7] L. Chakhmakhchyan and N. J. Cerf, Boson sampling with Gaussian measurements, Phys. Rev. A 96, 032326 (2017).

[8] M. Bentivegna, N. Spagnolo, C. Vitelli, F. Flamini, N. Viggianiello, L. Latmiral, P. Mataloni, D. J. Brod, E. F. Galvão, A. Crespi, et al., Experimental scattershot boson sampling, Sci. Adv. 1, e1400255 (2015).

[9] H. Wang, J. Qin, X. Ding, M.-C. Chen, S. Chen, X. You, Y.-M. He, X. Jiang, L. You, Z. Wang, et al., Boson sampling with 20 input photons and a 60-mode interferometer in a $10^{14}$-dimensional Hilbert space, Phys. Rev. Lett. 123, 250503 (2019).

[10] A. Deshpande, A. Mehta, T. Vincent, N. Quesada, M. Hinsche, M. Ioannou, L. Madsen, J. Lavoie, H. Qi, J. Eisert, et al., Quantum computational supremacy via high-dimensional Gaussian boson sampling, Preprint ArXiv:2102.12474 (2021).

[11] C. S. Hamilton, R. Kruse, L. Sansoni, S. Barkhofen, C. Silberhorn, and I. Jex, Gaussian Boson Sampling, Phys. Rev. Lett. 119, 170501 (2017).

[12] R. Kruse, C. S. Hamilton, L. Sansoni, S. Barkhofen, C. Silberhorn, and I. Jex, Detailed study of Gaussian boson sampling, Phys. Rev. A 100, 032326 (2019).

[13] W. R. Clements, J. J. Renema, A. Eckstein, A. A. Valido, A. Lita, T. Gerrits, S. W. Nam, W. S. Kolthammer, J. Huh, and I. A. Walmsley, Approximating vibronic spectroscopy 
with imperfect quantum optics, J. Phys. B: At., Mol. Opt. Phys. 51, 245503 (2018).

[14] S. Paesani, Y. Ding, R. Santagati, L. Chakhmakhchyan, C. Vigliar, K. Rottwitt, L. K. Oxenløwe, J. Wang, M. G. Thompson, and A. Laing, Generation and sampling of quantum states of light in a silicon chip, Nat. Phys. 15, 925 (2019).

[15] H.-S. Zhong, L.-C. Peng, Y. Li, Y. Hu, W. Li, J. Qin, D. Wu, W. Zhang, H. Li, L. Zhang, et al., Experimental Gaussian boson sampling, Sci. Bull. 64, 511 (2019).

[16] H.-S. Zhong, H. Wang, Y.-H. Deng, M.-C. Chen, L.-C. Peng, Y.-H. Luo, J. Qin, D. Wu, X. Ding, Y. Hu, et al., Quantum computational advantage using photons, Science 370, 1460 (2020).

[17] H.-S. Zhong, Y.-H. Deng, J. Qin, H. Wang, M.-C. Chen, L.-C. Peng, Y.-H. Luo, D. Wu, S.-Q. Gong, H. Su, et al., Phase-programmable Gaussian boson sampling using stimulated squeezed light, Preprint ArXiv:2106.15534 (2021).

[18] J. Arrazola, V. Bergholm, K. Brádler, T. Bromley, M. Collins, I. Dhand, A. Fumagalli, T. Gerrits, A. Goussev, L. Helt, et al., Quantum circuits with many photons on a programmable nanophotonic chip, Nature 591, 54 (2021).

[19] J. Huh, G. G. Guerreschi, B. Peropadre, J. R. McClean, and A. Aspuru-Guzik, Boson sampling for molecular vibronic spectra, Nat. Photonics 9, 615 (2015).

[20] J. M. Arrazola and T. R. Bromley, Using Gaussian Boson Sampling to Find Dense Subgraphs, Phys. Rev. Lett. 121, 030503 (2018).

[21] J. M. Arrazola, T. R. Bromley, and P. Rebentrost, Quantum approximate optimization with Gaussian boson sampling, Phys. Rev. A 98, 012322 (2018).

[22] L. Banchi, M. Fingerhuth, T. Babej, C. Ing, and J. M. Arrazola, Molecular docking with Gaussian boson sampling, Sci. Adv. 6, eaax1950 (2020).

[23] M. Schuld, K. Brádler, R. Israel, D. Su, and B. Gupt, Measuring the similarity of graphs with a Gaussian boson sampler, Phys. Rev. A 101, 032314 (2020).

[24] S. Jahangiri, J. M. Arrazola, N. Quesada, and N. Killoran, Point processes with Gaussian boson sampling, Phys. Rev. E 101, 022134 (2020).

[25] L. Banchi, N. Quesada, and J. M. Arrazola, Training Gaussian boson sampling distributions, Phys. Rev. A 102, 012417 (2020).

[26] S. Jahangiri, J. M. Arrazola, N. Quesada, and A. Delgado, Quantum algorithm for simulating molecular vibrational excitations, Phys. Chem. Chem. Phys. 22, 25528 (2020).

[27] A. Neville, C. Sparrow, R. Clifford, E. Johnston, P. M. Birchall, A. Montanaro, and A. Laing, Classical boson sampling algorithms with superior performance to near-term experiments, Nat. Phys. 13, 1153 (2017).

[28] P. Clifford and R. Clifford, in Proceedings of the TwentyNinth Annual ACM-SIAM Symposium on Discrete Algo- rithms (Society for Industrial and Applied Mathematics, 2018), New Orleans, Louisiana, p. 146.

[29] H. Ryser, Combinatorial Mathematics (The Mathematical Association of America, Rahway, New Jersey, 1963), Vol. 4.

[30] N. Quesada, J. M. Arrazola, and N. Killoran, Gaussian boson sampling using threshold detectors, Phys. Rev. A 98, 062322 (2018).

[31] B. Gupt, J. M. Arrazola, N. Quesada, and T. R. Bromley, Classical benchmarking of Gaussian boson sampling on the Titan supercomputer, Quantum Inf. Process. 19, 1 (2020).

[32] B. Wu, B. Cheng, F. Jia, J. Zhang, M.-H. Yung, and X. Sun, Charge-transport layer engineering in perovskite solar cells, Sci. Bull. 65, 1237 (2020).

[33] N. Quesada and J. M. Arrazola, Exact simulation of Gaussian boson sampling in polynomial space and exponential time, Phys. Rev. Res. 2, 023005 (2020).

[34] A. Björklund, B. Gupt, and N. Quesada, A faster hafnian formula for complex matrices and its benchmarking on a supercomputer, J. Exp. Algorithmics 24, 11 (2019).

[35] C. Weedbrook, S. Pirandola, R. García-Patrón, N. J. Cerf, T. C. Ralph, J. H. Shapiro, and S. Lloyd, Gaussian quantum information, Rev. Mod. Phys. 84, 621 (2012).

[36] A. Serafini, Quantum Continuous Variables: A Primer of Theoretical Methods (CRC Press, Boca Raton, FL, 2017).

[37] B. Picinbono, Second-order complex random vectors and normal distributions, IEEE Trans. Signal Process. 44, 2637 (1996).

[38] N. Quesada, L. G. Helt, J. Izaac, J. M. Arrazola, R. Shahrokhshahi, C. R. Myers, and K. K. Sabapathy, Simulating realistic non-Gaussian state preparation, Phys. Rev. A 100, 022341 (2019).

[39] K. Husimi, Some formal properties of the density matrix, Proc. Phys.-Math. Soc. Jpn. 3rd Ser. 22, 264 (1940).

[40] J. Williamson, On the algebraic problem concerning the normal forms of linear dynamical systems, Am. J. Math. 58, 141 (1936).

[41] M. M. Wolf, G. Giedke, O. Krüger, R. Werner, and J. I. Cirac, Gaussian entanglement of formation, Phys. Rev. A 69, 052320 (2004).

[42] J. E. Gentle, Computational Statistics (Springer, Heidelberg, 2009), Vol. 308.

[43] B. Gupt, J. Izaac, and N. Quesada, THE WALRUS: A library for the calculation of hafnians, hermite polynomials and Gaussian boson sampling, J. Open Source Softw. 4, 1705 (2019).

[44] R. Rehman and I. C. Ipsen, La Budde's method for computing characteristic polynomials, Preprint ArXiv:1104.3769 (2011).

[45] T.-W. Huang, D.-L. Lin, Y. Lin, and C.-X. Lin, TASKFLOW: a lightweight parallel and heterogeneous task graph computing system, Preprint ArXiv:2004.10908 (2020). 